San Jose State University

SJSU ScholarWorks

Master's Theses

Master's Theses and Graduate Research

Fall 2015

\title{
The Role of Nitric Oxide in Skeletal Simulated Microgravity Response
}

Luan Tran

San Jose State University

Follow this and additional works at: https://scholarworks.sjsu.edu/etd_theses

\section{Recommended Citation}

Tran, Luan, "The Role of Nitric Oxide in Skeletal Simulated Microgravity Response" (2015). Master's Theses. 4669.

DOI: https://doi.org/10.31979/etd.w92s-dmy3

https://scholarworks.sjsu.edu/etd_theses/4669

This Thesis is brought to you for free and open access by the Master's Theses and Graduate Research at SJSU ScholarWorks. It has been accepted for inclusion in Master's Theses by an authorized administrator of SJSU ScholarWorks. For more information, please contact scholarworks@sjsu.edu. 


\title{
THE ROLE OF NITRIC OXIDE IN SKELETAL SIMULATED MICROGRAVITY RESPONSE
}

\author{
A Thesis \\ Presented to \\ The Faculty of the Department of Biological Sciences \\ San José State University
}

In Partial Fulfillment

of the Requirements for the Degree

Master of Science

By

Luan H. Tran

December 2015 
(C) 2015

Luan H. Tran

ALL RIGHTS RESERVED 
The Designated Thesis Committee Approves the Thesis Titled

THE ROLE OF NITRIC OXIDE IN SKELETAL SIMULATED

MICROGRAVITY RESPONSE

by

Luan H. Tran

APPROVED FOR THE DEPARTMENT OF BIOLOGICAL SCIENCES

SAN JOSÉ STATE UNIVERSITY

December 2015

Dr. Ruth Globus

NASA-Ames Research Center

Dr. Daniel Holley

Department of Biological Sciences

Dr. Shelley Cargill

Department of Biological Sciences 


\title{
ABSTRACT \\ THE ROLE OF NITRIC OXIDE IN SKELETAL SIMULATED MICROGRAVITY RESPONSE
}

\author{
by Luan H. Tran
}

Prolonged exposure to the space environment leads to bone loss. Using a groundbased model for microgravity (hind-limb unloading), it has been shown in rodents that proliferation and differentiation activity of osteoprogenitors are decreased with exposure to simulated microgravity (Kostenuik et al., 1997). Osteoblast mineralization activity has been shown to increase with nitric oxide supplementation (Afzal et al., 2004; Koyama et al., 2000). I hypothesized that the degenerative effects of microgravity exposure result from chronic changes in nitric oxide regulation in osteoprogenitors, and that supplementation with exogenous nitric oxide following exposure would ameliorate these changes. In $20 \mathrm{C} 57 \mathrm{Bl} / 6$ mice, hind-limb unloading for up to 16 days resulted in degeneration of cancellous tissue in hind-limb bones and a decrease in inducible nitric oxide synthase (iNOS) gene expression in both bone tissue and bone marrow cells. Ex vivo analyses of osteoprogenitor cells, however, showed no changes in differentiation potential or cell count, and supplementation with sodium nitroprusside (SNP) was not able to increase mineralization activity in hind-limb unloaded mice. It is concluded that exogenous nitric oxide supplementation is ineffective as a countermeasure to the effects of simulated microgravity exposure on bone cells. 


\section{ACKNOWLEDGEMENTS}

I would like to express my deepest gratitude to my committee, Dr. Holley, Dr. Globus, and Dr. Cargill, for their guidance and the patience they have shown throughout this work. To Dr. Holley, who I have to thank for this amazing opportunity and whose direction, good spirit, and patience, carried me through to this accomplishment. To Dr. Globus, who has been a mentor to me since the beginning of my scientific career and whose guidance and support molded me into a capable researcher, allowing me to realize my potential. To Dr. Cargill, who has been a role model of diligence and dependability and whose advice helped me navigate through the confusion and complexity of conducting this research.

To the faculty and staff of the Department of Biological Sciences, particularly Larry Young, Dr. Bros, Dr. Parr, Dr. VanHoven, and Dr. Soto, whose efforts and advice demonstrate the quality and sincerity of the department's members and made me feel supported and important as a student. To the members of the Bone and Signaling Laboratory at the NASA Ames Research Center, especially Dr. Schruers, Dr. Alwood, Dr. Shanazari, Tiffany Truong, Kevin Bruns, and others, without whose contributions and encouragement this work would not have been possible.

Finally, to my friends and family, whose proud support and words of reassurance made every late night, long day, and unforeseen challenge worthwhile. 


\section{TABLE OF CONTENTS}

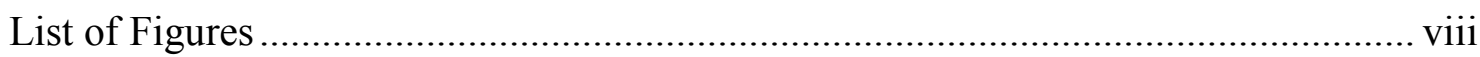

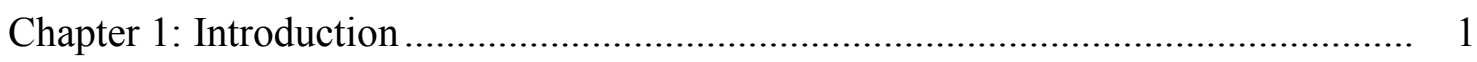

Bone Structure and Tissue ..................................................................... 2

Effects of Microgravity on Bone Structure...................................................... 3

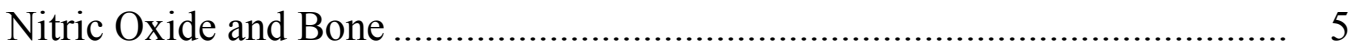

Chapter 2: Methodology ........................................................................... 6

Sample Acquisition and IACUC Approval .................................................. 6

In Vivo Response to Simulated Microgravity …………………………......... 7

Ex Vivo Osteoprogenitor Response to Simulated Microgravity ....................... 8

Nitric Oxide Supplementation as a Countermeasure …………………........... 8

Micro Computed Tomography of Bone........................................................... 9

Primary Culture of Bone Marrow Cells............................................................ 10

RNA Analyses of Bone and Bone Cells ........................................................ 11

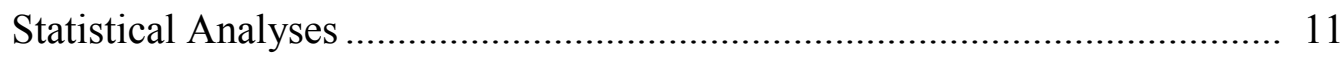

Chapter 3: Research Results …………………………..................................... 12

In Vivo Responses to Simulated Microgravity................................................ 12

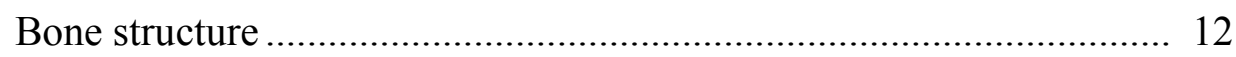

Gene expression ..................................................................... 13

Ex Vivo Osteoprogenitor Response to Simulated Microgravity ....................... 14

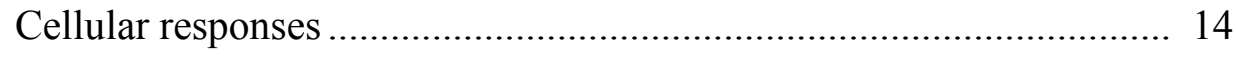

Nitric Oxide Supplementation as a Countermeasure ……................................ 16 
Cellular responses .......................................................... 16

Gene expression .................................................................. 17

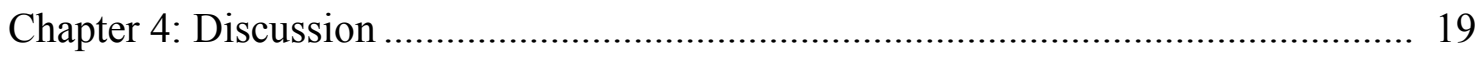

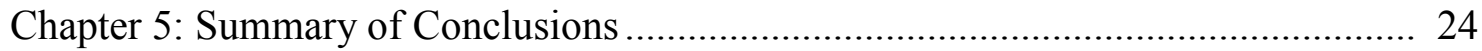

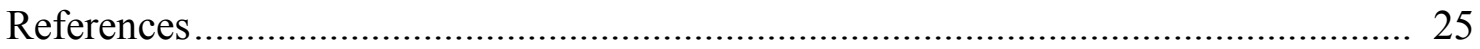




\section{LIST OF FIGURES}

\section{FIGURE}

1. Inducible nitric oxide synthase (iNOS) and endothelial nitric oxide synthase (eNOS) gene expression levels in femora of normally loaded (NL) and hind-limb unloaded (HU) C57Bl/6 mice after 16 days of unloading.

2. Inducible nitric oxide synthase (iNOS) and endothelial nitric oxide synthase (eNOS)gene expression levels in hind-limb bone marrow cells (BMC) of normally loaded (NL) and hind-limb unloaded (HU) C57Bl/6 mice after 16 days of unloading

3. p-nitrophenyl phosphate (pNPP) digestion by alkaline phosohatase from cell cultures of normally loaded (NL) and hind-limb unloaded (HU) C57B1/6 mice after 10 days of unloading and 7 days into culture.

4. DNA content of cell cultures from normally loaded (NL) and hind-limb unloaded (HU) C57B1/6 mice after up to 10 days of unloading and 3, 7, and 10 days of ex vivo cell culture

5. p-nitrophenyl phosphate (pNPP) digestion by alkaline phosohatase from cell cultures of hind-limb unloaded C57Bl/6 mice after 14 days of unloading .........

6. Growth area mineralized (\%) in cell cultures from hind-limb unloaded C57B1/6 mice after 14 days of unloading and 28 days of culture

7. Gene expression levels in cells from normally loaded (NL) and hind-limb unloaded (HU) C57B1/6 mice after 14 days of unloading and 9 days of ex vivo cell culure 


\section{Introduction}

Long-duration space travel and exposure to the space environment poses many risks to human health (Dunbar et al., 2013). Disuse osteoporosis is a condition of bone loss resulting from decreased mechanical loading and it is suspected that the free fall experienced in low earth orbit, termed microgravity, causes the observed degeneration of bone tissue - on average a loss of up to two percent per month while in low earth orbit (Erikson, 2011). Because of the rate of bone loss observed in the space environment, understanding bone responses to disuse is at the forefront of spaceflight health research.

There are two major challenges when addressing disuse osteoporosis in the space environment: 1) an incomplete understanding of molecular response mechanisms and 2) lack of simple, effective countermeasures. This study seeks to address these challenges by answering the following research question:

What role do nitric oxide generation and metabolism play in osteoprogenitor response to microgravity exposure? More specifically, is the degeneration of bone during microgravity exposure a result of impaired bone formation mediated by persistent but reversible changes in nitric oxide generation in osteoblast progenitors?

I hypothesize that microgravity exposure inhibits osteoblast differentiation by decreasing intracellular nitric oxide synthase expression in osteoblast progenitors. More specifically, simulated microgravity for up to 14 days will be sufficient to cause persistent decreases in nitric oxide synthase expression levels which will translate into impaired osteoblast progenitor differentiation ex vivo, and these changes can be mitigated 
by the introduction of a nitric oxide donor drug.

\section{Bone Structure and Tissue}

Bone is a dynamic tissue composed of many cell types which are relevant to health and normal body function. For example, bone marrow is a crucial source not only for mesenchymal stem cells (Jones et al., 2002), but also for signaling cells which have been reported to secrete bioregulators that interact during inflammatory responses (Grano et al., 2002). In addition to its physiological contribution, bone also provides structural support for the various organ systems. Bone tissue is made up of three main cell types, osteoblasts, osteoclasts, and osteocytes, each which have its own roles in bone health and kinetics. Osteoblasts are responsible for building bone, osteoclasts for resorbing, and osteocytes are critical for intercellular signaling and communication. Together these cell types resorb and rebuild bone, modeling it to meet the structural needs of its environment. Because the balance between osteoblasts and osteoclasts is so critical to bone health, any changes to vitality or function of a cell type may lead to a pathological phenotype of reduced bone mass if osteoblast activity is decreased relative to osteoclast activity, or abnormally increased bone density if osteoblast activity is increased relative to osteoclast activity. Since this study will focus mainly on structural changes and osteoblast responses as a metric for bone health, a brief summary of the two follows.

Bone tissue can generally be categorized into two types: 1) dense, rigid cortical bone, or 2) porous, high turnover cancellous bone. Cortical bone is the dense tissue found in outer regions of bone and is made up of subunits known as osteons. An osteon is a 
cylindrical structure composed of osteocyte cells, enclosed in mineralized extracellular matrix, concentrically arranged around a central canal which houses a nourishing blood vessel, lymphatics, and nerves. Osteons typically run parallel to the long axis of bone. In contrast, cancellous bone is found in the inner regions of bone and is structurally very porous. Cancellous tissue is made up of trabeculae, rod shaped subunits which act as structural support (Sherwood, 2015). In both cases, changes to physical properties such as density, mass, count, or spacing can lead to significant structural compromise. Since trabecular bone has higher turnover rates, it is analyzed in greater detail in this study.

Osteoblast cells and their progenitors are derived from mesenchymal stem cells, and are considered to be specialized fibroblasts which are able to form bone through a process of calcium deposition in their extracellular matrices, referred to as mineralization (Kini et al., 2012; Noda, 2014). Osteoblast activity is dependent on a number of cell processes. However, it is well established that osteoblastogenesis, and subsequent bone formation, is strongly dependent on proliferation and differentiation. Osteoblast proliferation is a strong mediator of differentiation, since osteoblastogenesis is preceded and regulated by proliferation (Stein et al., 1989). This suggests that changes in proliferation behavior of osteoblast progenitors can propagate into changes in mineralization and bone formation, since the mineralization of bone is mediated mainly by differentiated osteoblast cells.

\section{Effects of Microgravity on Bone Structure}

As with most tissues in the body, bone tissue health and integrity are a result of 
both internal signals and external influences. Beginning with early development, bone health is observed to be highly dependent on external mechanical input (Carter et al., 1996). Like other tissues in the body, bone health and integrity remain dependent on mechanical input for the duration of an organism's life. This ongoing relationship is at the heart of the microgravity response in bone.

In bone, microgravity exposure manifests itself as a loss of mineral mass which leads to diminished structural integrity. These changes are observed in both cortical and cancellous regions of bones that are subjected to weight bearing forces at $1 \mathrm{G}$, (Keyak et al., 2009; Lang et al., 2004). A majority of what is understood about bone response to microgravity, however, has been elucidated from experiments utilizing the hind-limb unloading model for microgravity (E. Morey-Holton et al., 2005). It has been shown that tibiae from hind-limb unloaded mice undergo degeneration similar to that seen with spaceflight while additional analyses demonstrated that these changes originate at the microscopic level. For example, tibiae from mice which have been hind-limb unloaded for up to 15 days show significant decreases in weight and volume with subsequent research identifying decreases in trabecular thickness and mineral apposition rate as potential contributing factors (Egrise et al., 2003; Globus et al., 1986).

Examining this effect further, it has been shown that microgravity exposure changes bone remodeling at the cellular level. In simulated microgravity, osteoblasts cells show diminished ability to proliferate, differentiate, or form mineralized matrices-all vital functions for proper bone remodeling (Basso et al., 2005; Kostenuik et al., 1997). It is suspected that a microgravity environment deprives osteoblast cells and their 
progenitors of necessary mechanical input and thus inhibits signals which regulate bone formation. This idea is substantiated by studies which have observed osteoblast response to mechanical input or long duration bedrest, which has been likened to microgravity exposure, and demonstrated that changes in the mechanical environment are able to regulate bone cell signaling and gene expression (Kim et al., 2003; Liedert et al., 2006; Papachroni et al., 2009).

\section{Nitric Oxide and Bone}

Nitric oxide is a free radical gas which exists naturally in all vertebrates (Knowles et al., 1994). In animals, nitric oxide acts predominantly as a signaling molecule (bioregulator) with a variety of functions ranging from vessel homeostasis to paracrine and autocrine signaling. Nitric oxide is generated in the body via an enzyme, nitric oxide synthase (NOS), which has three isoforms, designated as induced (iNOS), endothelial (eNOS), and neuronal (nNOS). These isoforms are activated in different conditions: iNOS is typically activated in response to external stimulus while eNOS and nNOS are constitutively expressed in certain cell types. The process of nitric oxide generation in the cell begins with an L-arginine molecule that is oxidized via successive monooxygenation reactions, and ultimately generates a single L-citrulline molecule, and a molecule of nitric oxide (Knowles et al., 1994).

With regards to bone homeostasis, nitric oxide signaling and response has been linked to mechanotransduction in bone, with decreases up to 66 percent in bone-forming surface, mineral apposition rate, and bone forming rate in mechanically loaded tibiae 
treated with a nitric oxide synthase inhibitor (Turner et al., 1996). Furthermore, nitric oxide has been shown to be active in osteoblast signaling and response; osteoblasts both produce and are receptive to nitric oxide-related signals (Hukkanen et al., 1995; Ralston et al., 1994; Riancho et al., 2009). A number of osteoblast functions have been shown to be dependent on eNOS expression-eNOS knockout mice show inhibition of bone formation gene expression, proliferation, and nodule formation as well as diminished bone mineral density (Afzal et al., 2004).

This study investigated the relationship between nitric oxide regulation in bone tissue and its responses to microgravity exposure. Additionally, the potential of a nitric oxide-based countermeasure to microgravity-induced bone loss was explored using sodium nitroprusside (SNP), a compound commonly used in research as an intracellular nitric oxide donor. This supplementation was provided to cell cultures from hind-limb unloaded mice in an attempt to nullify the inhibitory effects of microgravity.

\section{Methodology}

\section{Sample Acquisition and IACUC Approval}

All samples used in this study were acquired and processed according to protocol \# 2014-E, approved by the San Jose State University Institutional Animal Care and Use Committee. Although the following methods describe, for completeness, sample acquisition from live animals, all tissues and samples used for this study were independently generated and previously archived at the NASA Ames Research Center, and obtained subsequently as non-living tissue, either as preserved bone or as cell lysates. 


\section{In Vivo Response to Simulated Microgravity}

A total of 20 male C57B1/6 mice (Jackson West, Sacramento, CA) at 15 weeks of age were normally loaded or hind-limb unloaded for two weeks, according to a protocol developed by Morey-Holton et al. (2005), with minor adjustments made for adaptation to a mouse model ( $n=10$ group) (E. Morey-Holton et al., 2005; E. R. Morey-Holton \& Globus, 2002). At the end of the hind-limb unloading period, mice were euthanized and hind-limb bones were extracted, preserved in 4\% formaldehyde, and archived at the NASA Ames Research Center. Subsequent $\mu C T$ analyses were performed using a Skyscan 1174 high resolution microCT scanner (Bruker, Kontich, Belgium). Femora and tibiae were scanned, separately, at $6.7 \mu \mathrm{m}$ resolution for cancellous tissue analyses. For both femora and tibiae, analyses of tissue were targeted to proximal regions measured from the growth plate.

For gene expression analyses, an additional 20 male C57Bl/6 mice (Jackson West, Sacramento, CA) were either normally loaded or hind-limb unloaded for durations up to 16 days ( $n=10$ /group). After the unloading period, mice were euthanized via asphyxiation in $\mathrm{CO}_{2}$ chamber with subsequent cervical dislocation, and femora were extracted and flushed with minimum essential medium alpha (Gibco, Gaithersburg, MD) to clear the medullary canal of marrow fluid which was subsequently centrifuged to isolate bone marrow cells (BMC). BMC were archived as cell lysates at the NASA Ames Research Center, and subsequently acquired for this study. Gene expression analyses was performed via qRT-PCR (one-step RT-qPCR reaction kits, Promega, Madison, WI) with 
targeted markers for nitric oxide generation, inducible nitric oxide synthase and endothelial nitric oxide synthase (iNOS and eNOS).

\section{Ex Vivo Osteoprogenitor Response to Simulated Microgravity}

There were a total of 8 male C57B1/6 mice (Jackson West, Sacramento, CA) with half $(n=4)$ hind-limb unloaded for up to ten days and the remaining half $(n=4)$ subject to normal loading conditions for the same duration. After the unloading period, mice were euthanized via asphyxiation in a $\mathrm{CO}_{2}$ chamber with subsequent cervical dislocation, and BMC collected for primary culture in a total of eight plates. After 7 days of culture, adherent cells were preserved and analyzed for alkaline phosphatase enzymatic activity via Alkaline Phosphatase Activity Colorimetric Assay Kit (BioVision, Mountain View, CA). After 3, 7, and 10 days of culture, cells were preserved and analyzed for total DNA content via CyQUANT cell proliferation assay (Molecular Probes, Eugene, OR). Cell lysates were independently generated at the NASA Ames Research Center, archived, and subsequently acquired for this study.

\section{Nitric Oxide Supplementation as a Countermeasure}

A total of 10 male C57B1/6 mice (Jackson West, Sacramento, CA) were hind-limb unloaded for 14 days, after which hind-limb BMC were extracted for primary culture, in a total of 10 plates, either with or without sodium nitroprusside supplementation (Sigma, St. Louis, MO) at a final concentration of $0.5 \mathrm{uM}$ and using water as a vehicle. After 10 and 14 days of culture, adherent cells were lysed and analyzed for alkaline phosphatase enzymatic activity via Alkaline Phosphatase Activity Colorimetric Assay Kit (BioVision, 
Mountain View, CA). At 28 days of culture, cells were lysed using RLT buffer (Qiagen, Venlo, Netherlands) supplemented with $\beta$-mecaptoethanol to a final concentration of $1 \%$, and the mineralized matrix was stained for mineral using silver nitrate (Fisher, Fremont, CA).

For gene expression analyses via qRT-PCR (one-step RT-qPCR reaction kits, Promega, Madison, WI), 10 male C57Bl/6 mice were either normally loaded $(n=5)$ or hind-limb unloaded $(n=5)$ for four days. After the unloading period, hind-limb BMC were collected for primary culture either with or without sodium nitroprusside supplementation at a final concentration of $0.5 \mathrm{uM}$ in a total of 20 plates. After nine days in culture, adherent cells were lysed and assayed for expression levels of genes related to differentiation (ALP, BGLAP) and proliferation (p21). All cell lysates and cleared matrix were independently generated at the NASA Ames Research Center, archived, and subsequently acquired for this study.

\section{Micro Computed Tomography of Bone}

Hind-limb bones were dissected and cut either at the epiphyses, in femora, or at the tibia-fibula junction, in tibiae, to allow for the perfusion of 5\% paraformaldehyde (Sigma, St. Louis, MO) for 24 hours at $4^{\circ} \mathrm{C}$ and subsequent storage in $70 \%$ ethanol (Sigma, St. Louis, MO). Bones were scanned, in phosphate buffered saline (Gibco, Gaithersburg, MD), using a Skyscan 1174 high resolution microCT scanner (Bruker, Kontich, Belgium) at $6 \mu \mathrm{m}^{3}$ voxel resolution. In femur, the volume of interest was defined as a $1.2 \mathrm{~mm}$ thick region located $0.06 \mathrm{~mm}$ proximal to the distal growth plate. In tibia, the 
volume of interest was defined as a $1 \mathrm{~mm}$ thick region located $0.24 \mathrm{~mm}$ distal to the proximal growth plate. Cortical and cancellous bone tissues were differentiated semiautonomously, using the endocortical surface as a guide. Structural analyses were performed for the bone volume to total volume fraction (BV/TV, \%), trabecular thickness (Tb.Th, $\mu \mathrm{m})$, trabecular number (Tb.N, 1/mm), and trabecular separation (Tb.Sp, $\mu \mathrm{m})$ according to conventional guidelines (Bouxsein et al., 2010).

\section{Primary Culture of Bone Marrow Cells}

Marrow cells were flushed from bone using minimum essential media alpha (Gibco, Gaithersburg, MD) and purified of red blood cells via five-minute incubation in lysis buffer (Sigma-Aldrich, St. Louis, MO), centrifugation for ten minutes at $1000 \mathrm{x}$ g, and decanting of supernatant. Cells were then re-suspended and plated at $3.0 \times 10^{5}$ cells/ $\mathrm{cm}^{2}$ in either 6-well plates, for ALP, DNA, and qPCR assays, or in T-12.5 flasks for mineralization analyses. Growth media was composed of minimum essential media alpha (Gibco, Gaithersburg, MD) supplemented with 15\% fetal calf serum (Gibco, Gaithersburg, MD), ascorbate (50 $\mu \mathrm{g} / \mathrm{mL}$, Sigma-Aldrich, St. Louis, MO), and $\beta$ glycerophosphate (10 mM, Sigma-Aldrich, St. Louis, MO). Media was replenished every three days until termination of the experiment. Subsequent quantification of either ALP enzymatic activity or DNA content were performed using Alkaline Phosphatase Activity Colorimetric Assay (Biovision, Mountain View, CA) or CyQuant Cell Proliferation Assay Kit (Molecular Probes, Eugene, OR), respectively. For mineralization quantification, cells were allowed to grow in culture for 28 days, with media replenished every three days. Mineralized nodules were washed with phosphate buffered saline 
(Gibco, Gaithersburg, MD) and then with deionized water. Plates were then incubated in $20 \%$ bleach for five minutes and then stained with silver nitrate (Fisher, Fremont, CA) for 30 minutes and rinsed with deionized water. Scanning of plates was achieved using a Perfection 1640SU desktop scanner (Epson, Lo Beach, CA) set to 12000dpi resolution and resulting images were digitized and analyzed using ImageJ (National Institutes of Health, Public Domain).

\section{RNA Analyses of Bone and Bone Cells}

RNA was extracted from bone tissue or cell lysates via RNeasy Mini Kit (Qiagen,

Venlo, Netherlands). In the case of bone tissue, a preceding TRIzol (Invitrogen, Carlsbad, CA) protocol was performed according to manufacturer direction to pre-isolate samples for improved yield and quality. After being evaluated for quality via Nanodrop (ThermoFisher, Waltham, MA) and Bioanalyser (Agilent Technologies, Santa Clara, CA), samples were individually processed for qRT-PCR analyses using one-step RTqPCR reaction kits (Promega, Madison, WI). Gene expression was evaluated using the delta-delta CT method (Schmittgen et al., 2008) with genes of interest being normalized to a house-keeping gene, GAPDH.

\section{Statistical Analyses}

Statistical analyses were performed using JMP 10 (SAS Institute Inc, Cary, NC). For comparisons made only between normally loaded and hind-limb unloaded conditions, an unpaired t-test was used. For sodium nitroprusside supplementation experiments where NO supplementation was evaluated in conjunction with loading state, two-way 
ANOVA was performed. In all cases, figures report mean \pm standard deviation (SD) and $p \leq 0.05$ was considered the threshold for significance.

\section{Research Results}

\section{In Vivo Response to Simulated Microgravity}

Bone structure. Hind-limb unloading for two weeks was sufficient to induce significant changes in bone structure, as measured by $\mu \mathrm{CT}$. In cancellous tissue of tibiae, composed of trabeculae, both percent bone volume and trabecular thickness decreased significantly, by $17.9 \%$ and by $6.7 \%$ respectively, as compared to control mice (Table 1 ). In femora, hind-limb unloading for two weeks caused a $4.8 \%$ decrease in trabecular thickness, as compared to normally loaded controls.

Table 1

Effects of hind-limb unloading on bone structure of Male C57Bl/6 mice

Normally Loaded Hind-limb Unloaded
(2wks) (2wks)

\begin{tabular}{lll}
\hline Femora & & \\
Percent Bone Volume $(\%)$ & $18.6 \pm 2.8$ & $16.6 \pm 2.6$ \\
Trabecular Thickness $(\mu \mathrm{m})$ & $0.061 \pm 0.001$ & $0.058 \pm 0.001^{*}$ \\
Trabecular Number $(1 / \mathrm{mm})$ & $3.07 \pm 0.42$ & $2.88 \pm 0.43$ \\
Trabecular Separation $(\mu \mathrm{m})$ & $0.18 \pm 0.015$ & $0.19 \pm 0.013$ \\
& & \\
Tibiae & & $17.4 \pm 3.3^{*}$ \\
Percent Bone Volume $(\%)$ & $21.2 \pm 3.3$ & $0.055 \pm 0.003^{*}$ \\
Trabecular Thickness $(\mu \mathrm{m})$ & $0.059 \pm 0.003$ & $3.10 \pm 0.53$ \\
Trabecular Number $(1 / \mathrm{mm})$ & $3.58 \pm 0.47$ & \\
\hline
\end{tabular}

t-test, Data are mean $\pm \mathrm{SD}, \mathrm{n}=10 /$ group, $* p$-value $<0.05$ 
Gene expression. In femoral bone tissue without marrow, up to 16 days of hindlimb unloading resulted in a 53\% decrease in iNOS expression levels with no significant changes to eNOS expression (Fig. 1). Similarly, in femoral bone marrow cells, up to 16 days of hind-limb unloading resulted in undetectable levels of iNOS expression with no changes in eNOS expression (Fig. 2)
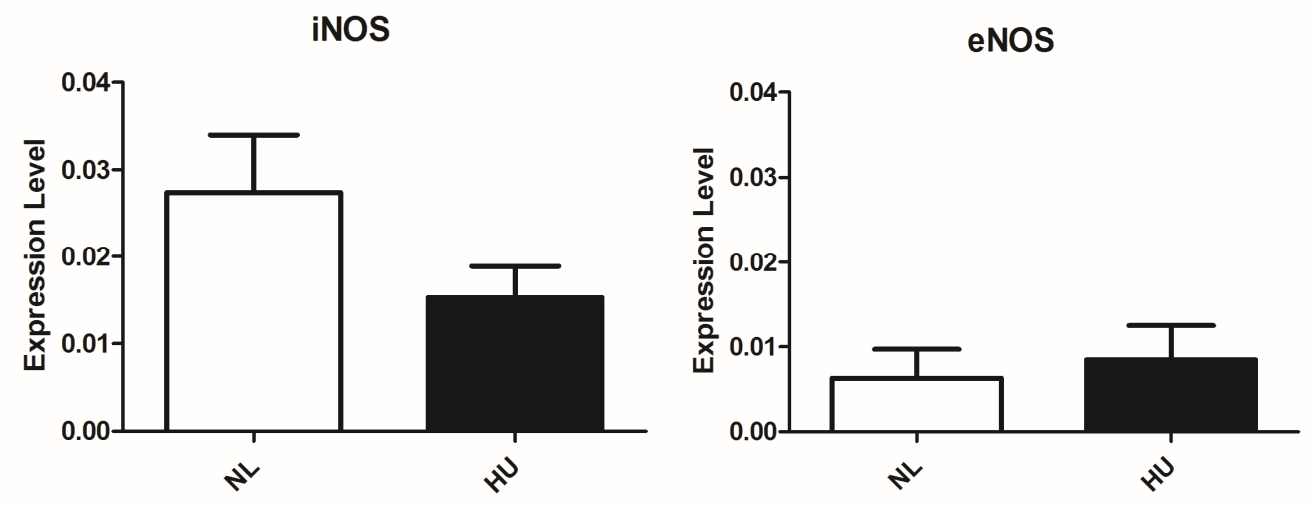

Figure 1. Inducible nitric oxide synthase (iNOS) and endothelial nitric oxide synthase (eNOS) gene expression levels in femora of normally loaded (NL) and hind-limb unloaded (HU) C57Bl/6 mice after 16 days of unloading. Expression levels normalized to housekeeping genes (GAPDH). Data are mean $+\mathrm{SD}, \mathrm{n}=10$ /group. $* p<0.05$. There were no significant differences in eNOS expression between NL and HU groups. 

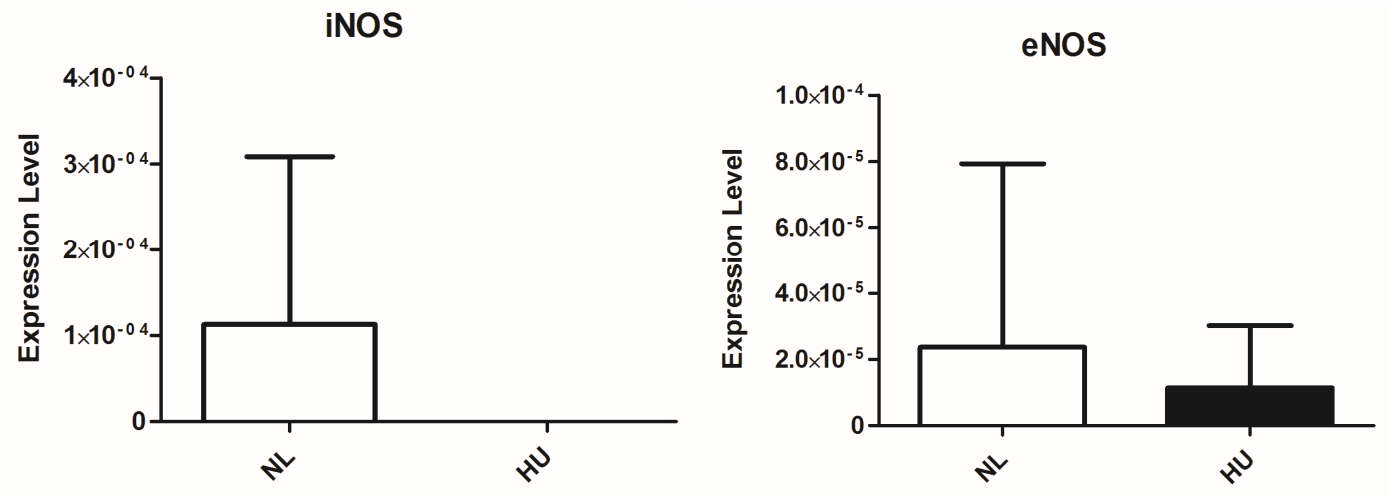

Figure 2. Inducible nitric oxide synthase (iNOS) and endothelial nitric oxide synthase (eNOS)gene expression levels in hind-limb bone marrow cells (BMC) of normally loaded (NL) and hind-limb unloaded (HU) C57B1/6 mice after 16 days of unloading. Expression levels normalized to a housekeeping gene (GAPDH). Data are mean + SD, $n=10$ /group. No significant differences were detected between NL and HU groups. There was no iNOS gene expression in HU derived cells.

\section{Ex Vivo Osteoprogenitor Response to Simulated Microgravity}

Cellular responses. After 7 days in culture, BMC from mice which were hind-

limb unloaded for ten days did not differ from controls with regards to alkaline phosphatase activity in osteoprogenitor cells (Fig. 3). Additional data show that hindlimb unloading for up to ten days was not able to change total DNA content, measured at three, seven, and ten days into culture (Fig.4). 
Day 7

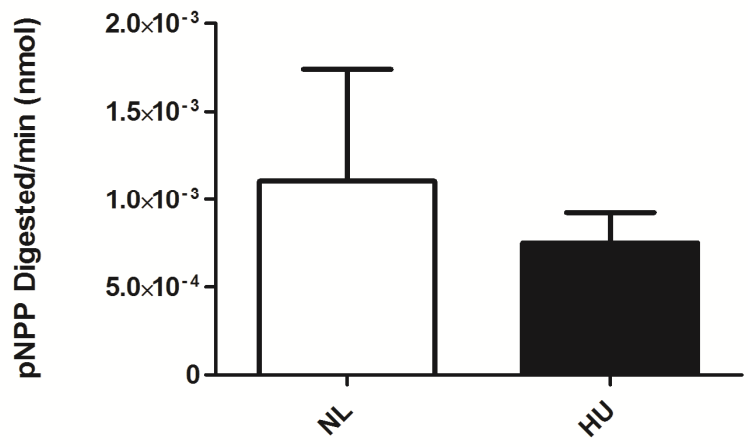

Figure 3. p-nitrophenyl phosphate (pNPP) digestion by alkaline phosohatase from cell cultures of normally loaded (NL) and hind-limb unloaded (HU) C57Bl/6 mice after 10 days of unloading and 7 days into culture $(n=4 /$ group). Values normalized to growth area. Data are mean \pm SD. No significant differences were detected between NL and HU groups.

A)

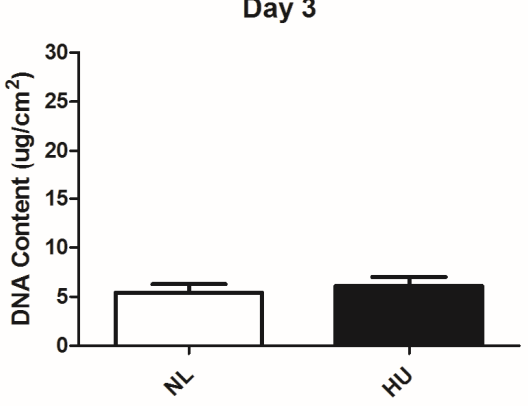

B)

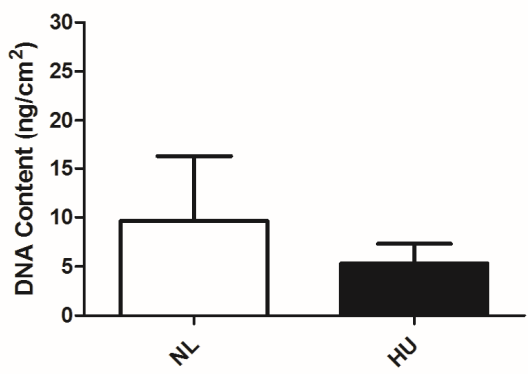

C)

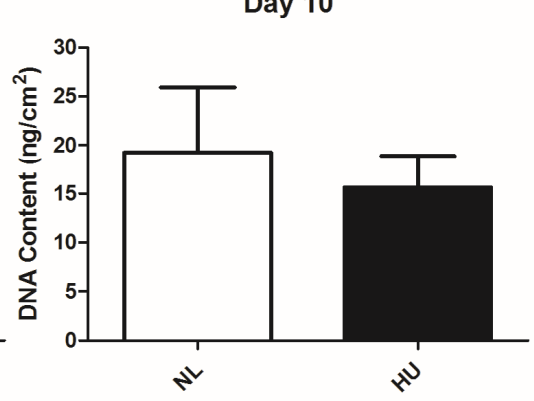

Figure 4. DNA content of cell cultures from normally loaded (NL) and hind-limb unloaded (HU) $\mathrm{C} 57 \mathrm{Bl} / 6$ mice after up to 10 days of unloading and 3, 7, and 10 days of ex vivo cell culture. Data are mean $\pm \mathrm{SD}, \mathrm{n}=4$ /group. No significant differences were detected between NL and HU groups. 


\section{Nitric Oxide Supplementation as a Countermeasure}

Cellular response. Results show that after two weeks of in vivo hind-limb unloading, ex vivo treatment of cultured marrow cells with sodium nitroprusside supplementation did not significantly alter ALP enzymatic activity either after 10 or 14 days of culture, compared to unsupplemented controls (Fig. 5). Additionally, no differences in total mineralization area were observed 28 days into culture between cells grown in standard growth media and cells grown in media supplemented with sodium nitroprusside to a final concentration of $0.5 \mathrm{uM}$ (Fig. 6).

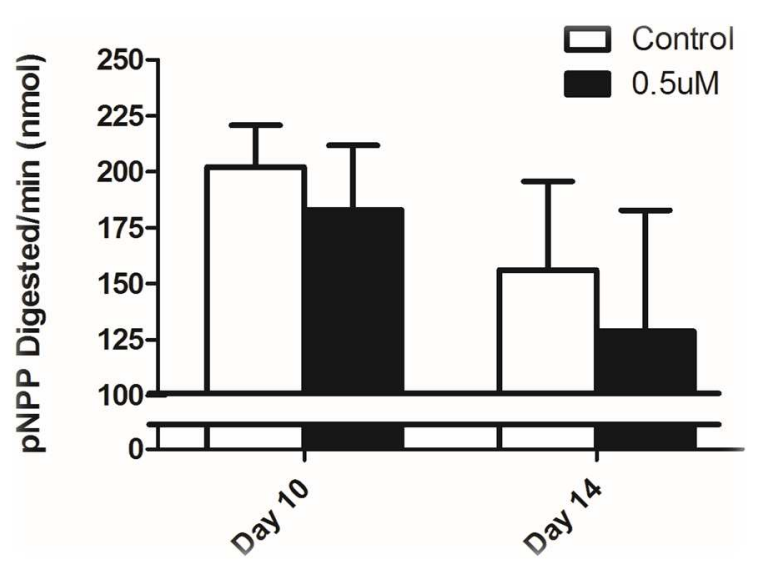

Figure 5. p-nitrophenyl phosphate (pNPP) digestion by alkaline phosohatase from cell cultures of hind-limb unloaded $\mathrm{C} 57 \mathrm{~B} 1 / 6$ mice after 14 days of unloading. Cells were cultured either in standard growth media (Control) or growth media supplemented with sodium nitroprusside $(0.5 \mathrm{uM})$. Values normalized to growth area. Data are mean $\pm \mathrm{SD}, \mathrm{n}=5$ /group. No significant differences were detected.

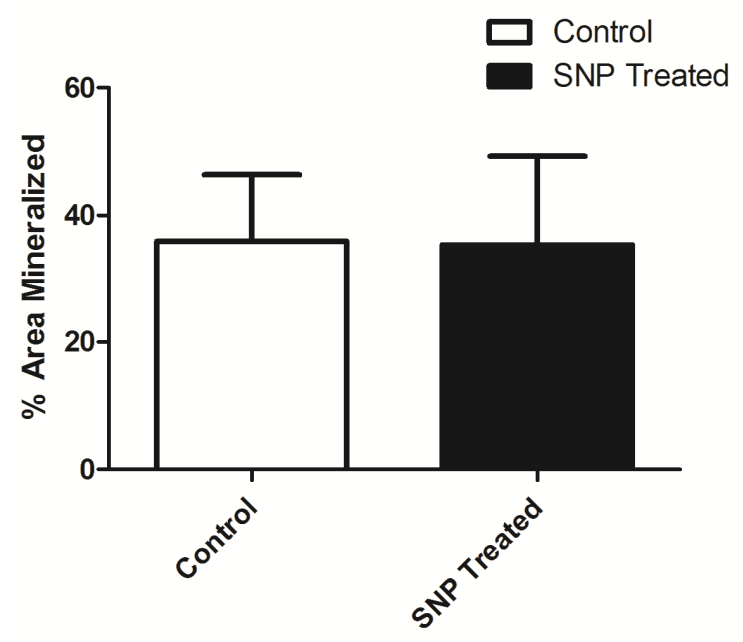

Figure 6. Growth area mineralized (\%) in cell cultures from hind-limb unloaded C57Bl/6 mice after 14 days of unloading and 28 days of culture. Cells were cultured either in standard growth media (Control) or growth media supplemented with sodium nitro-prusside to a final concentration of $0.5 \mathrm{uM}$ (SNP Treated). Data are mean $\pm \mathrm{SD}, \mathrm{n}=5$ /group. No significant differences were detected. 
Gene expression. Sodium nitroprusside supplementation was able to inhibit expression of differentiation gene BGLAP while simulated microgravity inhibited gene expression of $\mathrm{p} 21$. After nine days of growth, neither cultures from hind-limb unloaded animals nor cultures supplemented sodium nitroprusside showed any changes in ALP gene expression compared to normally loaded controls (Fig. 7A). In contrast, BGLAP expression was shown to be significantly decreased in cultures supplemented with sodium nitroprusside, regardless of loading status (Fig. 7B). With p21, expression was shown to be significantly increased in cultures from hind-limb unloaded mice, regardless of whether or not sodium nitroprusside was supplemented (Fig. 7C). 


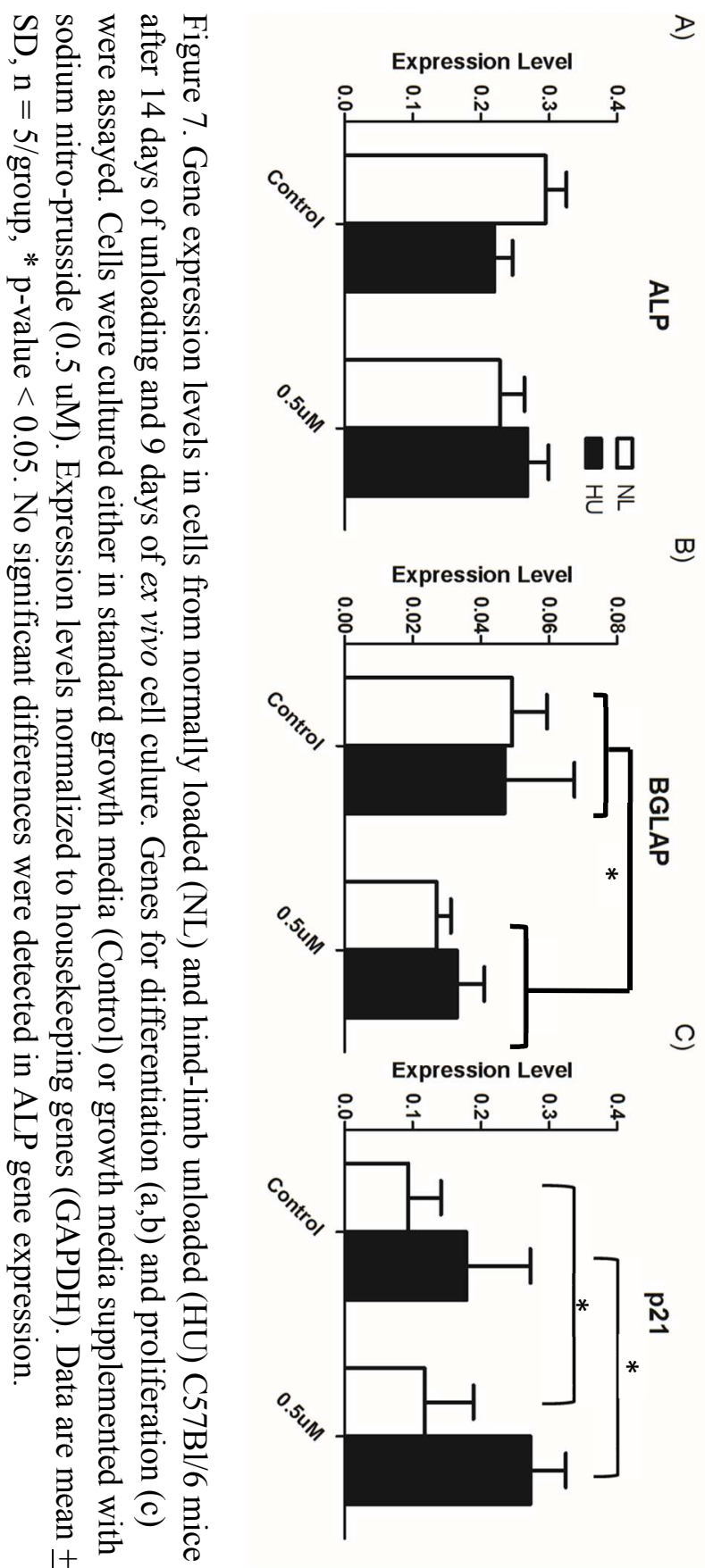




\section{Discussion}

\section{In Vitro Effects}

For this study, measures of bone structure and integrity were chosen based on parameters well-established in published literature. These included measures of overall bone volume, as well as structural characteristics of cancellous bone. Due to its importance in maintaining structural integrity, and because of its higher turnover rate, cancellous bone tissue is ideal for evaluation of structural responses.

Results indicate that two weeks of hind-limb unloading is sufficient to induce significant degeneration of bone tissue in femora and tibiae and is evident in key structural subunits of cancellous tissue, the trabeculae. This finding suggests a relationship between hind-limb unloading exposure and bone loss, but does not specify cellular or molecular mechanisms involved in this response.

Quantitative PCR was used to measure RNA expression levels of genes for nitric oxide generation in flushed bone tissue and in bone marrow cells, individually. Both inducible nitric oxide synthase (iNOS or NOS2) and endothelial nitric oxide synthase (eNOS or NOS3) gene expression were quantified for nitric oxide generation. Nitric oxide synthase is an integral enzyme in nitric oxide generation from L-arginine. Its product, nitric oxide (NO), is an important signaling molecule involved in a plethora of physiological functions, with osteoblast differentiation among them (Koyama et al., 2000; Ralston et al., 1994; Van'T Hof et al., 2001). iNOS, the inducible form of the enzyme, is known to generate high levels of NO in response to stimulus while eNOS, the endothelial form of the enzyme, provides a more consistent source of NO in the cell, 
constitutively expressed in normal tissues. Quantitative PCR was chosen to investigate the cellular responses in vivo because of its resolution and possible parallels to protein expression.

It was important to distinguish between bone tissue cells and bone marrow cells in order to distinguish the stromal and adherent cells of bone tissue, which contain osteoblast lineage cells, from the mostly haematopoietic cell populations of the marrow. Separation of bone marrow cells from bone tissue results in more specificity of the gene expression results. It was found that hind-limb unloading for up to 16 days was able to down-regulate expression of iNOS in both bone and marrow cells, but had no effect on eNOS expression in either bone or marrow. It should be noted that eNOS expression typically does not respond to external, environmental stimulation (Van'T Hof et al., 2001). Therefore, the inability of hind-limb unloading to induce changes in eNOS expression is not surprising. The hind-limb unloading induced inhibition of iNOS expression, however, suggests a correlation between hind-limb unloading and the nitric oxide metabolism potential of bone, and suggests that the changing levels of nitric oxide synthase expression are in response to an external stimulus.

Although gene expression is a highly reliable and informative method of analysis, it is not without its limitations. Changes in gene expression do not always result in changes to phenotype or molecular mechanisms or protein levels. Gene expression is only a single step in the regulation of cellular responses and may be encouraged or inhibited by other regulatory processes. Although mineralized bone tissue and bone marrow contain certain cell types, they are heterogeneous populations. As with any 
analysis performed on a heterogeneous population, single subpopulation effects may be masked by the whole, and may be unquantifiable using the methods employed in these experiments.

\section{Ex Vivo Effects}

To build upon the conclusions drawn from in vivo results, it was necessary to corroborate the findings with data from osteoprogenitors, specifically. During osteoblastogenesis, progenitor cells undergo a series of changes which lead to unique expression of genes and proteins. These changes coincide predictably with different stages of osteoblastogenesis and therefore can be used as indicators of differentiation state. Additionally, because osteoblastogenic activity is regulated by cell contact, proliferation (a prerequisite for establishing cell density) can be used to evaluate osteoblastogenic potential.

Measures of alkaline phosphatase activity were used to determine differentiation state of ex vivo osteoprogenitors, while DNA quantification was used as an estimate of cell number which eventually affects differentiation potential. Alkaline phosphatase removes phosphate groups from biological molecules and is commonly used as an indicator of early differentiation in osteoprogenitors, since its expression and activity increase at the onset of differentiation (Pittenger et al., 1999). DNA quantification is used as an indirect measure of cell count and, by evaluating changes in cell count over time, proliferation.

Since ALP activity was unchanged in differentiating osteoprgenitors, it is unlikely that the mechanism of effect influences the early stages of osteoblastogenesis in 
osteoprogenitors. This does not necessarily exclude potential effects manifested in later stages of osteoblast differentiation. With regards to cell number and proliferation, these findings suggest that simulated microgravity does not affect cell number or proliferation in osteoprogenitors.

\section{Nitric Oxide Supplementation as a Countermeasure}

Since nitric oxide has been shown to increase mineral deposition in osteoblasts (Afzal et al., 2004), a mechanism inhibited by simulated microgravity, I hypothesized that exogenous nitric oxide treatment of cells from hind-limb unloaded mice would counteract the inhibitory effects of hind-limb unloading in osteoprogenitors. Sodium nitroprusside is a compound commonly used in medicine as a vasodilator and in research as an intracellular nitric oxide donor. As a donor, sodium nitroprusside is more stable than its eventual breakdown product, nitric oxide. Because of this stability, it is able to be taken in by the cell before it breaks down, effectively increasing nitric oxide concentrations within the cell.

The efficacy of nitric oxide as a countermeasure to osteoprogenitor microgravity response was evaluated by observing changes in early differentiation, proliferation, and gene expression of osteoprogenitors as measured by ALP activity and qPCR following SNP supplementation in growth media. For qPCR analyses, p21, a gene which codes for a cyclin-dependent kinase inhibitor that down-regulates cell cycle progression was chosen as a biomarker for inhibition of proliferation. ALP and BGLAP are proteins expressed in early and late stages, respectively, of osteoblast differentiation and were chosen as biomarkers for differentiation state. ALP was chosen because alkaline 
phosphatase expression is observed to increase predictably at the onset of osteoblastogenesis while BGLAP, also known as osteocalcin, was chosen because of its positive correlation to bone mineral density and its origins as a product of differentiated osteoblasts.

Since SNP supplementation did not alter ALP activity in osteoprogenitors from hind-limb unloaded animals, I conclude that nitric oxide treatment did not affect early stages of osteoblast differentiation. This is not surprising considering that simulated microgravity does not influence ALP activity in normal cells. This was corroborated by gene expression data which indicated no differences in ALP gene expression between SNP supplemented groups and their respective controls. Interestingly, gene expression results suggest that both simulated microgravity and nitric oxide supplementation are able to affect osteoprogenitor function, although each in separate cellular mechanisms. Upregulation of p21 gene expression in cultures from hind-limb unloaded animals indicates that proliferation is inhibited with simulated microgravity exposure; a conclusion supported by several studies (Blaber et al., 2013; Dai, Wang et al., 2007; Hughes-Fulford et al., 1996). Alternatively, nitric oxide supplementation down-regulated BGLAP and not ALP or p21 gene expression in culture, implying that nitric oxide regulates later stages of differentiation and not earlier stages or proliferation. It is no surprise, then, that SNP supplementation was not able to improve mineralization ability of cells from hind-limb unloaded mice. 


\section{Summary and Conclusions}

This study shows that two weeks of simulated microgravity via hind-limb unloading is sufficient to induce gene expression responses which regulate nitric oxide metabolism in vivo in bone tissue, and associated stromal cells. Furthermore, cell culture analyses of bone stromal cells revealed that these changes remain persistent in osteoprogenitors after nine days in culture. Introduction of exogenous nitric oxide via sodium nitroprusside supplementation was not able to ameliorate the persistent effects of simulated microgravity nor able to improve mineralization capacity of previously hindlimb unloaded osteoprogenitors. The failure of exogenous nitroprusside to counteract the effects of simulated microgravity in osteoprogenitors may lie in the fact that it downregulates gene expression of differentiation marker BGLAP, whereas simulated microgravity exposure, in vivo, was found to upregulate proliferation gene $\mathrm{p} 21$ in ex vivo cell culture. This distinction signifies that the influences of microgravity and nitric oxide metabolism manifest in separate mechanisms of osteoblast regulation. This suggests that nitric oxide, despite its ability to regulate osteoblastogensis, is not a suitable countermeasure to microgravity-induced responses in osteoprogenitors. 


\section{References}

Afzal, F., Polak, J., \& Buttery, L. (2004). Endothelial nitric oxide synthase in the control of osteoblastic mineralizing activity and bone integrity. The Journal of Pathology, 202, 503-510.

Basso, N., Jia, Y., Bellows, C. G., \& Heersche, J. N. (2005). The effect of reloading on bone volume, osteoblast number, and osteoprogenitor characteristics: Studies in hind limb unloaded rats. Bone, 37, 370-378.

Blaber, Elizabeth A; Dvorochkin, Natalya; Lee, Chialing; Alwood, Joshua S; Yousuf, Rukhsana; Pianetta, Piero; Globus, Ruth K; Burns, Brendan P; Almeida, EA. (2013). Microgravity induces pelvic bone loss through osteoclastic activity, osteocytic osteolysis, and osteoblastic cell cycle inhibition by CDKN1a/p21. PLoS One, 8, e61372.

Bouxsein, M. L., Boyd, S. K., Christiansen, B. A., Guldberg, R. E., Jepsen, K. J., \& Müller, R. (2010). Guidelines for assessment of bone microstructure in rodents using micro-computed tomography. Journal of Bone and Mineral Research, 25, 14681486.

Carter, D., Van der Meulen, M., \& Beaupre, G. (1996). Mechanical factors in bone growth and development. Bone, 18, S5-S10.

Dai, Z., Wang, R., Ling, S., Wan, Y., \& Li, Y. (2007). Simulated microgravity inhibits the proliferation and osteogenesis of rat bone marrow mesenchymal stem cells. Cell Proliferation, 40, 671-684.

Dunbar, B., \& Roberts, J. (2013). How astronauts are affected by space exploration. Retrieved from http://www.nasa.gov/externalflash/HRP_Feature/

Egrise, D., Holy, X., Hinsenkamp, M., Begot, L., Schoutens, A., Bergmann, P., \& Zerath, E. (2003). Protracted systemic changes in bone biology after segmented unloading in the rat. Calcified Tissue International, 73, 56-65.

Erikson, K. (2011). Space bones. Retrieved from http://science.nasa.gov/sciencenews/science-at-nasa/2001/ast01oct_1/

Globus, R. K., Bikle, D. D., \& Morey-Holton, E. (1986). The temporal response of bone to unloading. Endocrinology, 118, 733-742. 
Grano, M; Mori, G; Minielli, V; Barou, O; Colucci, S; Giannelli, G; Alexandre, C; Zallone, AZ; Vico, L. (2002). Rat hindlimb unloading by tail suspension reduces osteoblast differentiation, induces IL-6 secretion, and increases bone resorption in ex vivo cultures. Calcified Tissue International, 70, 176-185.

Hughes-Fulford, M., \& Lewis, M. L. (1996). Effects of microgravity on osteoblast growth activation. Experimental Cell Research, 224, 103-109.

Hukkanen, Mika; Hughes, Francis J; Buttery, LDK; Gross, Steven S; Evans, TJ; Seddon, Shaun; Riveros-Moreno, Valnetina; Macintyre, Iain; Polak, Julia M. (1995).

Cytokine-stimulated expression of inducible nitric oxide synthase by mouse, rat, and human osteoblast-like cells and its functional role in osteoblast metabolic activity. Endocrinology, 136, 5445-5453.

Jones, E. A., Kinsey, S. E., English, A., Jones, R. A., Straszynski, L., Meredith, D. M., . . . McGonagle, D. (2002). Isolation and characterization of bone marrow multipotential mesenchymal progenitor cells. Arthritis \& Rheumatism, 46, 33493360 .

Keyak, J., Koyama, A., LeBlanc, A., Lu, Y., \& Lang, T. (2009). Reduction in proximal femoral strength due to long-duration spaceflight. Bone, 44, 449-453.

Kim, H., Iwasaki, K., Miyake, T., Shiozawa, T., Nozaki, S., \& Yajima, K. (2003). Changes in bone turnover markers during 14-day 6 head-down bed rest. Journal of Bone and Mineral Metabolism, 21, 311-315.

Kini, U., \& Nandeesh, B. (2012). Physiology of bone formation, remodeling, and metabolism. Radionuclide and hybrid bone imaging (pp. 29-57) Springer.

Knowles, R. G., \& Moncada, S. (1994). Nitric oxide synthases in mammals. The Biochemical Journal, 298 ( Pt 2)(Pt 2), 249-258. Retrieved from http://www.ncbi.nlm.nih.gov/pmc/articles/PMC1137932/pdf/biochemj000920010.pdf

Kostenuik, P. J., Halloran, B. P., Morey-Holton, E. R., \& Bikle, D. D. (1997). Skeletal unloading inhibits the in vitro proliferation and differentiation of rat osteoprogenitor cells. The American Journal of Physiology, 273, E1133-9.

Koyama, A., Otsuka, E., Inoue, A., Hirose, S., \& Hagiwara, H. (2000). Nitric oxide accelerates the ascorbic acid-induced osteoblastic differentiation of mouse stromal $\mathrm{ST} 2$ cells by stimulating the production of prostaglandin $\mathrm{E}<\mathrm{sub}>2$. European Journal of Pharmacology, 391, 225-231. 
Lang, T., LeBlanc, A., Evans, H., Lu, Y., Genant, H., \& Yu, A. (2004). Cortical and trabecular bone mineral loss from the spine and hip in Long-Duration spaceflight. Journal of Bone and Mineral Research, 19, 1006-1012.

Liedert, A., Kaspar, D., Blakytny, R., Claes, L., \& Ignatius, A. (2006). Signal transduction pathways involved in mechanotransduction in bone cells. Biochemical and Biophysical Research Communications, 349, 1-5.

Morey-Holton, E. R., \& Globus, R. K. (2002). Hindlimb unloading rodent model: Technical aspects. Journal of Applied Physiology, 92, 1367-1377.

Morey-Holton, E., Globus, R. K., Kaplansky, A., \& Durnova, G. (2005). The hindlimb unloading rat model: Literature overview, technique update and comparison with space flight data. Advances in Space Biology and Medicine, 10, 7-40.

Noda, M. (2014). Cellular and molecular biology of bone Academic Press.

Papachroni, K. K., Karatzas, D. N., Papavassiliou, K. A., Basdra, E. K., \& Papavassiliou, A. G. (2009). Mechanotransduction in osteoblast regulation and bone disease. Trends in Molecular Medicine, 15, 208-216.

Pittenger, M. F., Mackay, A. M., Beck, S. C., Jaiswal, R. K., Douglas, R., Mosca, J. D., . . . Marshak, D. R. (1999). Multilineage potential of adult human mesenchymal stem cells. Science (New York, N.Y.), 284, 143-147.

Ralston, S., Todd, D., Helfrich, M., Benjamin, N., \& Grabowski, P. (1994). Human osteoblast-like cells produce nitric oxide and express inducible nitric oxide synthase. Endocrinology, 135, 330-336.

Riancho, J. A., Salas, E., Zarrabeitia, M. T., Olmos, J. M., Amado, J. A., FernándezLuna, J. L., \& González-Macías, J. (2009). Expression and functional role of nitric oxide synthase in osteoblast-like cells. Journal of Bone and Mineral Research, 10, 439-446.

Schmittgen, T. D., \& Livak, K. J. (2008). Analyzing real-time PCR data by the comparative CT method. Nature Protocols, 3, 1101-1108.

Sherwood, L. (2015). Human physiology: From cells to systems Cengage learning.

Stein, S. G., Lian, B. J., Gerstenfeld, G. L., Victoria, S., Michael, A., Thomas, O., \& Elizabeth, M. (1989). The onset and progression of osteoblast differentiation is functionally related to cellular proliferation. Connective Tissue Research, 20, 3-13. 
Turner, C. H., Takano, Y., Owan, I., \& Murrell, G. (1996). Nitric oxide inhibitor LNAME suppresses mechanically induced bone formation in rats. American Journal of Physiology-Endocrinology and Metabolism, 270, E634-E639.

Van'T Hof, R. J., \& Ralston, S. H. (2001). Nitric oxide and bone. Immunology, 103, 255261. 